\title{
Validación en español del cuestionario específico para enfermedad respiratoria crónica autodiligenciado (CRQ-AUTOSELF)
}

\author{
Validation in Spanish of the self-administered Chronic \\ Respiratory Disease Questionnaire (CRQ-AUTOSELF)
}

\author{
Olga Chapeton Chaves, FT. ${ }^{(1)}$; Liliana Fernández T., MD. (2); William Martínez, MD. ${ }^{(3)}$; \\ FERNANDO SANABRIA, MD. ${ }^{(3)}$; JULIANA LENIS, FT. ${ }^{(4)}$; NANDY CASTRO, FT. ${ }^{(4)}$; \\ AlejandRo CASTILLO, MD., MSc. ${ }^{(5)}$
}

\section{Resumen}

INTRODUCCIÓN: el cuestionario de Calidad de Vida relacionada con salud CRQ (Chronic Respiratory Questionnaire) se cita en numerosos estudios que evalúan el impacto de las intervenciones no farmacológicas de la enfermedad pulmonar obstructiva crónica y requiere entrevistador con entrenamiento, lo cual demanda tiempo. La versión autoadministrada ha demostrado excelente validez y sensibilidad para detectar los cambios, comparado con otros instrumentos; pese a ser validada al castellano peninsular, los términos utilizados no son claros ni familiares para la población hispana.

OBJETIVO: validar al español hispanoamericano del CRQ autoadministrado, en una gama amplia de enfermedades pulmonares crónicas.

MATERIALES Y MÉTODOS: estudio de corte transversal de validación de una escala que contó con adaptación cultural y traducción de la versión original en inglés; para realizar la validación y el análisis factorial se utilizó el criterio de por lo menos 5 personas o participantes por cada ítem que tuviera el instrumento. En este caso, la escala CRQ tiene 20 ítems, para un total de 126 participantes. El análisis de datos se hizo en el programa STATA versión 10,0.

RESULTADOS: se incluyeron 126 pacientes que diligenciaron toda la escala. Se realizó el análisis factorial del instrumento; inicialmente se calculó el índice KMO, que se encontró en 0,92 , lo que hace concluir que la matriz obtenida es factorizable. Se realizó el análisis factorial por componentes principales y se extrajeron cuatro factores que explican el $71,1 \%$ de la varianza.

CONCLUSIONES: los resultados del estudio aportan evidencia acerca de la validez y confiabilidad de la escala en la versión autodiligenciada del CRQ en sujetos con enfermedad pulmonar obstructiva crónica y otras enfermedades pulmonares avanzadas.

Palabras clave: enfermedad pulmonar crónica, calidad de vida relacionada con salud, CRQ autodiligenciado, cuestionario.

\section{Abstract}

INTRODUCTION: the Chronic Respiratory Questionnaire is mentioned in many studies that assess the impact of non-pharmacologic interventions on chronic obstructive pulmonary disease. It requires a trained interviewer, which demands time. The self-administered version has shown excellent validity and sensitivity to detect changes, compared to other instruments. Although it has been validated in the Spanish spoken in Spain, the terms used are not clear or familiar to the Latin American population.

OBJECTIVE: to validate in Latin American Spanish the self-administered CrQ on a wide variety of chronic pulmonary diseases.

(1)Fisioterapeuta, Especialista en Fisioterapia Cuidado Crítico Adulto, Jefe Servicio de Terapia Respiratoria y Rehabilitación Pulmonar, Fundación Valle del Lili. Docente cátedra Universidad del Valle, Especialización en Fisioterapia Cardiopulmonar, Docente Cátedra Universidad CES, Especialización Cuidado Crítico Adulto. Cali, Colombia.

${ }^{(2)}$ Especialista en Medicina Interna y Neumología, Neumología Intervencionista, Línea del Investigación Biomédica en Tórax, Fundación Valle del Lili. Profesora Clínica Asociada, Facultad de Ciencias de la Salud, Universidad Icesi. Cali, Colombia.

${ }^{(3)}$ Especialista en Medicina Interna y Neumología, Línea del Investigación Biomédica en Tórax, Fundación Valle del Lili. Profesor Clínico Asistente, Facultad de Ciencias de la Salud, Universidad Icesi. Cali, Colombia.

${ }^{(4)}$ Fisioterapeuta, Especialista en Actividad Física Terapéutica, Fisioterapeuta Rehabilitación Pulmonar y coordinadora de Fisioterapia Programa Trasplante Pulmonar, Fundación Valle del Lili. Cali, Colombia

${ }^{(5)}$ Fisioterapeuta, Especialista en Rehabilitación Cardiopulmonar, Fisioterapeuta Rehabilitación Pulmonar, coordinadora de Fisioterapia Programa de Fibrosis Quística. Fundación Valle del Lili. Cali, Colombia ${ }^{(6)}$ Especialista en Psiquiatría, Maestría en Epidemiología, Fundación Valle del Lili. Profesor Clínico Asistente, Facultad de Ciencias de la Salud, Universidad Icesi. Cali, Colombia.

Correspondencia: Olga Chapeton Chaves, correo electrónico: ochapeton@fcvl.org Recibido: 20/02/16. Aceptado: 01/03/16. 
MATERIALS AND METHODS: cross-cut study for validating a scale with cultural adaptation and translation from the original English version; in order to carry out the validation and the factor analysis, a criterion of at least 5 persons or participants per item of the instrument was used. In this case, the CrQ has 20 items, for a total number of 126 participants. Data analysis was done with the 10,0 version of the StAtA program.

RESULTS: a total number of 126 patients who filled the complete scale were included. Factor analysis of the instrument was carried out; the KMO index was first calculated, and found to be 0,92 , which leads to the conclusion that the obtained matrix is factorizable. The factor analysis by main components was carried out; four factors were obtained, which explains the $71,1 \%$ variance.

CONCLUSIONS: the results of the study give evidence about the validity and reliability of the scale in the self-administered version of the CrQ in subjects with chronic obstructive pulmonary disease and other advanced pulmonary diseases.

Keywords: chronic pulmonary disease, health-related quality of life, self-administered CrQ, questionnaire.

\section{Introducción}

El estudio de las relaciones entre salud y calidad de vida en los pacientes que padecen enfermedades respiratorias crónicas con un grado elevado de discapacidad, debe estar guiado por una evaluación lo más objetiva posible y orientado al paciente con un enfoque de intervención multidisciplinar. Este tipo de intervenciones y abordajes facilita la comparación de los diferentes tipos de entrenamiento físico, estrategias educativas y automanejo $(1,2)$.

El cuestionario de enfermedad respiratoria crónica (CRQ) fue desarrollado en la Universidad de McMaster como una prueba de medición clínica para dar respuesta a la relación que existe entre la capacidad al ejercicio y la función pulmonar (3). Sus autores, Guyatt et al. generaron datos acerca de la situación de los pacientes y las limitaciones en las actividades de la vida diaria por su enfermedad, especialmente por la obstrucción de la vía aérea. Los estudios reportaron buenas características de sensibilidad, confiabilidad y especificidad.

El CRQ inicialmente fue construido para que fuera diligenciado por un entrevistador, y en su versión original está dividido en cuatro áreas o dimensiones: disnea, fatiga, función emocional y control de la enfermedad (4). Para disnea se identifican las 5 primeras preguntas, en fatiga las preguntas $8,11,15,17$, en la función emocional las preguntas $6,9,12,14,16,18,20 \mathrm{y}$ finalmente para control de la enfermedad las preguntas 7, 10, $13 \mathrm{y}$ 18. Los puntajes para cada dimensión se realizan por promedios y se usa uno máximo de 7 puntos en la escala de Likert.

En general, los cuestionarios específicos se centran en dominios que tienen una relevancia mayor para ciertas enfermedades, con el objetivo de describir las alteraciones de la calidad de vida de los pacientes. Los más utilizados en pacientes con enfermedad respiratoria y cuadros obstructivos crónicos en estudios de investigación, incluyen el CRQ The Chronic Respiratory Disease, SEPAR Insuficiencia respiratoria y SAOS, St. George's Respiratory Questionnaire (SGRQ) (5). Estos se componen de ítems que relacionan aspectos relevantes del control, grados de disnea, compromiso emocional y social. Para su aplicación la mayoría requiere de un entrevistador entrenado. Sin embargo, lo ideal es disponer de cuestionarios de salud más breves, que simplifiquen la evaluación y tengan correlación adecuada con las herramientas más complejas y validadas (6). Los autodirigidos tienen la ventaja de necesitar menos tiempo dedicado, pero requieren que la población estudiada tenga competencia en lecto-escritura de modo que favorezca las respuestas confiables; por ejemplo está el Airways Questionnaire 20 (AQ20), desarrollado por Quirk y Jones (6), que es autoadministrado, breve y específico, para pacientes con cuadros respiratorios obstructivos.

El CRQ autodirigido, es un cuestionario específico para la medición de la calidad de vida de personas con enfermedad pulmonar crónica; está diseñado para ser desarrollado por el paciente, con 
asistencia de un administrador solo si es necesario (7). Dado que cada ítem que incluye el cuestionario refleja las áreas de función, el paciente debe identificar con mínima orientación los aspectos que para él representan impedimento en las diferentes categorías y así no sesgar el resultado.

En vista del incremento en las remisiones a los servicios de Rehabilitación Pulmonar de enfermedades diferentes a la EPOC, nuestro interés fue validar el CRQ autodiligenciado en el idioma español (en hispanoamérica) y ofrecer un instrumento específico que aplique a cualquier enfermedad pulmonar avanzada. Si bien ya está validado en español, algunos términos utilizados en la validación del castellano peninsular, suscitan confusión en nuestro lenguaje a pesar de la reproducibilidad, sensibilidad al cambio e impacto que muestran los estudios donde se cita (8). Pensando en la optimización del recurso humano, el tiempo invertido en los cuestionarios administrados por el personal de salud usualmente es mínimo, aproximadamente media hora, dato que hace atractivo tener la opción validada del CRQ autodiligenciado para aplicar en individuos que cumplan los criterios de inclusión que abarcaron los pacientes del estudio.

El propósito de este estudio fue validar el cuestionario al idioma español, en población colombiana (CRQ-SR) en un grupo de pacientes sometidos a rehabilitación pulmonar.

\section{Metodología}

Se realizó un estudio de corte transversal de validación de una escala, que se aplicó a todos los participantes de la muestra en un momento único. Se llevó en la Santiago de Cali, en población clínica de adultos referidos al Programa de Rehabilitación Pulmonar de la Fundación Valle del Lili, que aceptaron participar y tenían competencia en lecto-escritura.

Inicialmente se hizo una adaptación cultural de la versión original en inglés de la escala a través de un proceso de traducción - retrotraducción y la realización de entrevistas cognitivas. Para tal fin, se conformó un comité de revisión de la misma, constituido por ocho personas: dos médicos especialistas con experiencia en el manejo de la enfermedad pulmonar y/o en el manejo de escalas y el grupo de Rehabilitación Pulmonar. Los dos médicos involucrados tienen como lengua nativa el idioma español, y fluencia en inglés (bilingües). Realizaron un proceso de traducción directa del inglés al español, con la indicación de hacer énfasis en el sentido conceptual de cada uno de los ítems. Después de la traducción se compararon las dos versiones en relación con el significado de los ítems de interés.

Pasada una semana se hizo una traducción inversa de la escala (traducción al inglés) de la versión que había sido traducida al español por el otro médico especialista participante, para establecer una equivalencia conceptual entre las dos versiones e identificar las diferencias importantes entre los dos traductores.

Para el cálculo de tamaño de la muestra en la validación y la realización del análisis factorial se utilizó el criterio de por lo menos 5 personas o participantes por cada ítem que tuviera el instrumento. En el caso de la escala CRQ, el número de ítems es 20 , para 100 participantes, más $20 \%$ de no respuesta, para un total de 120 participantes.

Se elaboró una base de datos con control de ingreso, a través de reglas de validación de los valores introducidos en el formulario para garantizar la confiabilidad de los datos (programa Excel), posteriormente se llevó al programa STATA versión 10.0, en el que finalmente se efectuó el análisis de la información.

Se llevó a cabo un análisis exploratorio de los datos, se evaluó la normalidad de su distribución y se identificaron valores extremos que pudieran incidir en el resultado, al igual que valores perdidos. Posteriormente, se hizo un análisis descriptivo de la población sujeto de estudio de acuerdo con las variables definidas. Para validar el contenido a fin de determinar los factores subyacentes, se realizó un análisis factorial por componentes principales. Inicialmente se aplicó el test de Kaiser-Meyer-Olkin (KMO), que es una medida de la suficiencia 
de la muestra. Posteriormente se hizo un análisis con los autovalores originales, para decidir el número de factores a extraer y se siguieron los criterios mencionados en la literatura: autovalor mayor a uno, conocida como la regla de Kaiser-Guttman y varianza explicada por el factor mayor al $5 \%$.

Se hicieron rotaciones ortogonal y oblicua por el método de Varimax y de Oblimin, respectivamente, y se compararon las matrices obtenidas en aras de comparar los dos métodos y decidirse por una matriz con "estructura simple", es decir que la hiciera más interpretable. Para establecer la consistencia interna se calculó el coeficiente de alfa de Cronbach para los ítems de la escala y adicionalmente para cada uno de los factores.

\section{Resultados}

Se incluyeron y valoraron 148 pacientes de los cuales el $51,3 \%$ fueron hombres, con edad promedio de 64,2 años (DS: 15,6); se incluyeron personas entre 15 y 91 años. Los hombres fueron mayores (65,1 años) que las mujeres (63,1 años) (tabla 1).

Tabla 1. Características socio demográficas.

\begin{tabular}{|lcc|}
\hline $\begin{array}{l}\text { Características } \\
\text { sociodemográficas }\end{array}$ & $\begin{array}{c}\text { Frecuencia } \\
\mathbf{n = 1 4 8}\end{array}$ & Porcentaje \\
Sexo & 76 & 51,3 \\
Masculino & 72 & 48,7 \\
Femenino & 148 & 100 \\
Total & & \\
Tabaquismo & 34 & 22,9 \\
Si activo & 117 & 80,1 \\
\hline No o exfumadores & & \\
\hline
\end{tabular}

\begin{tabular}{|lrrr|}
\hline \multicolumn{1}{|c}{ Edad } & $\begin{array}{c}\text { Grupo total } \\
\mathbf{n = 1 4 8}\end{array}$ & $\begin{array}{c}\text { Masculino } \\
\mathbf{n = 7 6}\end{array}$ & $\begin{array}{c}\text { Femenino } \\
\mathbf{n = 7 2}\end{array}$ \\
Promedio & 64,2 años & 63,1 años $^{*}$ & 65,1 años* \\
Desviación estándar & 15,6 & 14,9 & 16,3 \\
Mediana & 67 años & 64 años & 68,5 años \\
Rango & $15-91$ años & 30-91 años & $15-89$ años \\
\hline
\end{tabular}

${ }^{*} p$ valor: 0,43 (t test).

\begin{tabular}{|lccc|}
\hline \multicolumn{1}{|c}{ Paquetes laño } & $\begin{array}{c}\text { Grupo total } \\
\mathbf{n = 4 4}\end{array}$ & $\begin{array}{c}\text { Masculino } \\
\mathbf{n = 3 3}\end{array}$ & $\begin{array}{c}\text { Femenino } \\
\mathbf{n = 1 1}\end{array}$ \\
\hline Promedio & 31,7 & 35,3 & 25,8 \\
Desviación estándar & 13,9 & 14,6 & 10,8 \\
Mediana & 30 & $40^{*}$ & $23^{*}$ \\
Rango & $10-65$ & $12-65$ & $10-50$ \\
\hline
\end{tabular}

${ }^{*} p$ valor: 0,07 ( $K$ Wallis). 
En relación con los antecedentes médicos, 53 $(35,8 \%)$ tenían diagnóstico de EPOC y 92 reportaron otras enfermedades pulmonares asociadas (tabla 2).

De la muestra, 126 individuos diligenciaron toda la escala, información con la cual se hizo el análisis factorial de la misma. Inicialmente se calculó el índice KMO, que se encontró en 0,92 , lo que permitió concluir que la matriz obtenida es factorizable. Se realizó el análisis factorial por componentes principales y se extrajeron cuatro factores que explican el $71,1 \%$ de la varianza. Con la matriz inicial se probó una rotación de Varimax, pero dado que se encontró correlación entre los factores se prefirió

Tabla 2. Antecedentes médicos.

\begin{tabular}{|c|c|c|}
\hline Enfermedad pulmonar & $\begin{array}{l}\text { Frecuencia* } \\
\qquad n=148\end{array}$ & Porcentaje* \\
\hline EPOC & 53 & 35,8 \\
\hline Fibrosis pulmonar & 18 & 12,2 \\
\hline $\begin{array}{l}\text { Cáncer pulmonar o } \\
\text { metastásico a pulmón }\end{array}$ & 9 & 6,1 \\
\hline Asma & 5 & 3,4 \\
\hline Escleroderma & 5 & 3,4 \\
\hline Post neumonía & 5 & 3,4 \\
\hline Lobectomía pulmonar & 5 & 3,4 \\
\hline Bronquiectasias & 4 & 2,7 \\
\hline $\begin{array}{l}\text { Tromboembolia } \\
\text { pulmonar }\end{array}$ & 4 & 2,7 \\
\hline Hipertensión pulmonar & 4 & 2,7 \\
\hline Empiema torácico & 3 & 2 \\
\hline $\begin{array}{l}\text { Posoperatorio cirugía } \\
\text { pared de tórax }\end{array}$ & 3 & 2 \\
\hline $\begin{array}{l}\text { Tuberculosis pulmonar - } \\
\text { Postoperatorio }\end{array}$ & 3 & 2 \\
\hline artritis reumatoide & 2 & 1,4 \\
\hline Fibrosis quística & 2 & 1,4 \\
\hline $\begin{array}{l}\text { Enfermedad pulmonar } \\
\text { Intersticial }\end{array}$ & 2 & 1,4 \\
\hline Otras & 21 & 14,2 \\
\hline
\end{tabular}

la rotación de Promax, con lo que se obtuvo una matriz de mejor interpretación (tabla 3).

En el factor 4 solo cargaron las preguntas 9, 10 y 13; sin embargo las números 13,14 y 18 cargan en más de un factor, en las diferentes soluciones exploradas.

Al evaluar la confiabilidad se encontró un alfa de Cronbach para los factores 1, 2, 3 y 4 de 0,93, $0,89,0,83$ y 0,70 , respectivamente

\section{Discusión}

Se encontró una escala CRQ con cuatro factores o dominios, que coincide con los resultados del estudio original de validación de la escala; disnea, fatiga, función emocional y control de la enfermedad (4). Para disnea se identifican las 5 primeras preguntas, pero para los demás dominios de la escala el estudio factorial en esta población colombiana muestra diferencias con relación a la escala original en algunas de las pregunta, sugiere que la escala se comporta de manera diferente en la población del estudio y confirma la necesidad de este estudio de validación.

En 2011 se publicó la primera validación al español del CRQ autodirigido identificado en el estudio como CRQ-SAS (estandarizado al castellano peninsular), en el que los autores reportaron buenas propiedades tanto discriminativas como evaluativas para la medida de la calidad de vida relacionada con salud (CVRS) en pacientes con EPOC y concluyeron que la versión española del CRQ-SAS resulta ser válida para evaluar la CVRS de los pacientes con dicha enfermedad.

La CVRS es un componente de amplio concepto definido como la percepción del individuo en términos de satisfacción con su salud actual en el contexto cultural donde se desenvuelve desde lo físico, funcional, psicológico y social. Es por esto que los dominios del estado de salud se han subdividido en subdominios mucho más concretos (8) cuando de evaluar la CVRS se trate; esto facilita valorar aspectos que no son estrictamente clínicos sino relacionados con la vida diaria y cómo se ve afectada por la enfermedad de base. 
Tabla 3. Coeficiente de alfa de Cronbach.

\begin{tabular}{|cccccc|}
\hline Ítem & Factor 1 & Factor 2 & Factor 3 & Factor 4 & Uniqueness \\
p1 & & 0,8946 & 0,2198 \\
p2 & & 0,8537 & 0,2263 \\
p3 & & 0,8401 & 0,2196 \\
p4 & & 0,4605 & 0,6030 \\
\hline p5 & & 0,6946 & 0,3880 \\
p6 & 0,9468 & & & 0,2500 \\
p7 & 0,8170 & 0,3909 & & 0,2393 \\
p8 & & & & 0,4597 \\
p9 & & & 0,8822 & 0,2300 \\
p10 & & & 0,3993 & 0.3917 \\
p11 & & 0,7834 & & 0.2545 \\
p12 & 0,8781 & & & 0,2394 \\
p13 & & 0,3596 & 0.3489 & 0,3034 \\
p14 & 0,4953 & 0,4410 & & 0,3029 \\
p15 & & 0,9101 & & 0,2091 \\
p16 & 0,7323 & & & 0,2210 \\
p17 & & 0,8376 & & 0,1917 \\
p18 & 0,4740 & 0,5536 & & 0,3170 \\
p19 & 0,5259 & & & 0,3146 \\
p20 & 0,8249 & & & 0,1812 \\
\hline
\end{tabular}

En individuos sintomáticos con EPOC, independiente del grado de limitación al flujo aéreo, que participan en Rehabilitación Pulmonar obtienen resultados positivos al disminuir los síntomas, aumentar la tolerancia al ejercicio y mejorar la calidad de vida relacionada con salud, ventajas trasladables a otras enfermedades respiratorias avanzadas diferentes a la EPOC.

En la literatura existen instrumentos genéricos que son considerados menos discriminativos y sensibles al cambio, y que se aplican a la población general (9), además de los cuestionarios específicos para la enfermedad pulmonar crónica.

El interés al realizar este estudio fue hacer la validación en Colombia del cuestionario Chronic
Respiratory Disease Questionnaire (CRQ) en la versión autodirigida dada la sensibilidad al cambio que ha mostrado con la intervención de la rehabilitación pulmonar, pues ha servido como guía para la modulación del tratamiento médico, ha favorecido aún más la evidencia sobre el impacto en la calidad de vida relacionada con salud de la rehabilitación pulmonar y ha quedado como opción aplicable en países latinoamericanos que comparten semejanzas culturales y de lenguaje con las nuestras.

Como se indicó al principio del documento, cabe reiterar que existe una validación al español de este mismo instrumento con terminología cultural común de la península que en muchos apartados no es fácil de entender en nuestro medio. 
En relación con los resultados de confiabilidad de la escala, se encontró un alfa de Cronbach de 0,93 , que indica un resultado satisfactorio, sin embargo, puede también interpretarse que la escala (CRQ) tiene ítems redundantes, particularmente en el factor que evalúa la disnea.

Los resultados de este estudio aportan evidencia acerca de la validez y confiabilidad de la escala CRQ en la versión autoaplicable en personas con enfermedad pulmonar crónica, tanto EPOC como diferentes a esta. El hecho de no requerir evaluador la hace rápida y fácil de administrar y por ende reduce el tiempo de evaluación y los costos $(6,11)$.

\section{Conflictos de interés}

Los autores declaran no tener conflictos de interés.

\section{Bibliografía}

1. Spruit MA, Singh SJ, Garvey C, et al. An Official American Thoracic Society/European Respiratory Society Statement: Key Concepts and Advances in Pulmonary Rehabilitation. Am J Respir Crit Care Med. 2013;188:e29-35.

2. Singh SJ, ZuWallack RL, Garvey C, et al. Learn from the past and create the future: the 2013 ATS/ERS statement on pulmonary rehabilitation. Eur Respir J. 2013;42:1169-74.

3. Guyatt GH, Townsend M, Keller J, et al. Measuring functional status in chronic lung disease: conclusions from a randomised control trial. Respir Med. 1989;83:293-7.

4. Guyatt GH, Berman LB, Townsend M, et al. A measure of quality of life for clinical trials in chronic lung disease. Thorax. 1987;42:773-8.

5. Güell Rous MR, Díaz Lobato S, Rodríguez Trigo G, Morante Vélez F, San Miguel M, et al. Rehabilitación respiratoria. Arch Bronconeumol. 2014;50(8): 332-44.

6. Williams JEA, Singh SJ, Sewell L Morgan MDL. Health status measurement: sensitivity of the self-reported Chronic Respiratory Questionnaire (CRQ-SR) in pulmonary rehabilitatio. Thorax. 2003;58;515-8.

7. Jones PW. Health status measurement in chronic obstructive pulmonary disease. Thorax. 2001;56:880-7.

8. Vigil L, Güell MR, Morante F, López De Santamaría E, Sperati $F$, Guyatt $D$, et al. Validez y sensibilidad al cambio de la versión española autoadministrada del cuestionario de la enfermedad respiratoria crónica (CRQ-SAS). Arch Bronconeumol. 2011;47(7):343-9.

9. Lacasse Y, Wong E, Guyatt GH, et al. Meta-analysis of respiratory rehabilitation in chronic obstructive pulmonary disease. Lancet. 1996;348:1115-9.

10. Boueri FM, Bucher-Bartelson BL, Glenn KA, Make BJ. Quality of life measured with a generic instrument (Short Form 36) improves following pulmonary rehabilitation in patients with COPD. Chest. 2001;119:77-84.

11. Puhan MA, Behnke M, Frey M, Grueter T, Brandli O, Lichtenschopf A, et al. Self-administration and interviewer administration of the German Chronic Respiratory Questionnaire: instrument development and assessment of validity and reliability in two randomized studies. Health Qual Life Outcomes 2004;8(2):1.

12. Doyle C, Dunt D, Ames D, Selvarajah S. Managing mood disorders in patients attending pulmonary rehabilitation clinics. Int J of COPD. 2013;8:15-20. 